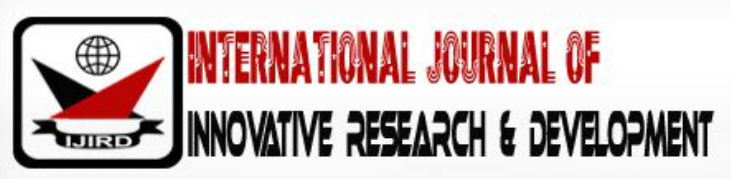

ISSN 2278 - 0211 (Online)

\section{Health Education and Disease Outbreak Control in Nigeria}

\author{
Ekenedo, Golda. 0. \\ Lecturer, Department of Human Kinetics and Health Education, University of Port Harcourt, Nigeria
}

\begin{abstract}
:
The Nigerian society has dealt with series of old and emerging disease outbreaks even in the recent past decade. This paper explored the role of health education in the control of disease outbreak in the country with particular reference to adult health education. The concepts of disease outbreak and disease prevention and control were discussed. Adequate health education methods for providing health education for the control of disease outbreaks were identified and discussed while the role of Information and Communication Technology in driving the process effectively was succinctly exposed. Finally, constraints to effective health education for disease control in Nigeria which included dearth of professional health educators, low level computer literacy and lack of infrastructure were discussed. It was concluded that controlling disease outbreak within a population cannot be effectively achieved without education interventions. Based on the conclusion, suggestions for mitigating the identified constraints were offered including that all health professionals who work in health facilities including should receive appropriate training in health education so that they can effectively carry out health education functions especially during disease outbreaks.
\end{abstract}

Keywords: Health education, information technology, disease outbreak, disease control, adult

\section{Introduction}

The place of education in human development cannot be argued and research continues to document the significant roles education play in solving various life problems in the areas of science and technology, health, agriculture, economics and the rest. In fact, it would have been impossible for the world to make any serious advancement in any field of life without the instrument of education. Health is one area where the role of education becomes so vivid. Health is a significant and most important aspect of life without which optimal function is not possible. Education stands out as a major public health promotion strategy and forms the basic essence of disease prevention. Knowledge they say is power, and knowledge that comes from sound education empowers individuals to make informed decisions concerning their health and that of others which are capable of preventing diseases and promoting health.

Diseases and ill-health have been with man since the beginning of time. Ironically, as breakthroughs are achieved in the cure and eradication of some diseases new ones are emerging. Hence, the fight against diseases is a continuous one for man. Diseases sometimes occur in excess of expectations involving a large proportion of the population and spreading quickly through the population. At such times, we say that an outbreak has occurred, an emergency situation requiring quick and prompt actions. Disease outbreak has a lot of impact on individuals and nations resulting in loss of human and financial resources, hence, the need for quick intervention. Actions in the control of disease outbreaks require multi-disciplinary approach from the areas of medical, paramedical, information and communication technology, education, environmental health, micro-biology, epidemiology, non-governmental agencies and so on. However, many researchers and authorities have affirmed that education, whether at individual, group or community level, remains key in diseases outbreak prevention and control.

\section{The Concept of Disease and Disease Outbreak}

Disease is a condition in which the body health is impaired, a departure from a state of health, an alteration of the human body, interrupting the performance of vital functions. Williamson, and Carr, (2009) defined disease as a condition of the body or some part or organ of the body in which the functions are disrupted or damaged. Diseases are classified into two major forms, communicable and non-communicable diseases. Communicable diseases are diseases that are transferable from one person or animal or object to another either through direct contact or through a vehicle. They are caused by microorganisms also known as germs. Examples of communicable diseases are malaria, cholera, tuberculosis, typhoid fever, Ebola virus, and Lassa fever. Non-communicable diseases are diseases that are not transferable. The causes of non-communicable diseases are largely unknown. They develop and progress slowly and last for a long period of time. As a matter of fact they are 
not curable but can be managed, and most importantly, prevented. Examples of non-communicable diseases include cancers, diabetes, cardiovascular diseases (diseases of the heart and blood vessels), arthritis, Alzheimer's disease and organ failures. A disease outbreak is the occurrence of cases of disease in excess of what would normally be expected in a defined community, geographical area or season (WHO, 2017). WHO explained that an outbreak may occur in a restricted geographical area, or may extend over several countries, and may last for a few days or weeks, or for several years. Public Health England - PHE (2014) viewed disease outbreak as a greater than expected rate of infection compared with the usual background rate for the place and time where the outbreak has occurred. They also revealed that a single case for certain rare diseases such as diphtheria, botulism, rabies, viral haemorrhagic fever or polio is equally regarded as an outbreak. In other words, in such rare diseases as mentioned above, even a single case qualifies as an outbreak. In the management of disease outbreak, PHE (2014) noted that the primary objective is to protect public health by implementing control measures to prevent further spread or recurrence of the infection. Mass education, at such times, is very pivotal in curbing the spread of the disease and in reducing fatality rate.

There are some concepts that describe certain situations of disease outbreak which include endemic, epidemic, pandemic and sporadic. Endemic describes a disease that is always present in a population. It is constant, it is continuous, it is recurrent in a particular place; example, malaria. Epidemic is the occurrences of a disease which rapidly and simultaneously attack a large number of people in a particular locality, (it can be in a L.G.A or State etc.) e.g. Cholera. Pandemic describes a wide spread disease covering more or less the whole world at the same time. It is across the globe e.g. HIV and AIDS. Sporadic is a disease occurrence in isolated or single case. It is not endemic or epidemic in nature, that is, intermittent. Examples are certain coughs and conjunctivitis.

There have been recorded cases of disease outbreak in Nigeria and across the globe. For instance, in recent past, there have been outbreaks of Ebola disease in parts of African countries, Lassa fever and cerebrospinal meningitis (CSM) in Nigeria. Nigeria also witness sporadic outbreaks of Cholera, meningitis and conjunctivitis every year. Also, outbreaks can occur in a given place following exposure to certain chemicals or radioactive materials such as the soot being currently witnessed in parts of Rivers State.

\subsection{Concepts of Disease Control and Disease Prevention}

Disease control is a term used when there is an already existing disease. It consists of specific efforts or step taken to reduce disease occurrence (incidence and prevalence) to a tolerable level. According to Hedi (2015) Disease control is a reduction in the incidence, prevalence, morbidity or mortality of an infectious disease to a locally acceptable level: elimination as reduction to zero of incidence of disease or infection in a defined area: and eradication as permanent reduction to zero of the worldwide incidence of infection (WHO, 2006). By this definition, with respect to control and elimination, it appears that there is inherent need for continued intervention approaches to prevent re-emergence and re-establishment of transmission. It is, therefore, ongoing operations aimed at reducing: the incidence of disease; the duration of disease and consequently the risk of transmission; the effects of infection, including both the physical and psychosocial complications; and the financial burden to the community.

Disease prevention according to Yazachew and Alem (2004) is the planning for and the measures taken to forestall the onset of a disease or other health problem before the occurrence of undesirable health events. It tries to look at specific activities designed to protect people (patients or other members of the public) from actual or potential health threats and their possible harmful consequences. It can be said to be general term for any maneuver aimed at reducing the incidence or effects of disease. It involves inhibiting the introduction of disease into a given geographical area or individual. There are three basic levels of prevention thus: primary, secondary and tertiary prevention.

Primary prevention describes action taken prior to the onset of disease, which removes the possibility that the disease will ever occur. As Yazachew and Alem (2004) pointed out, it comprised of those activities carried out to keep people healthy and prevent them from getting disease. Primary prevention may be accomplished by measures designed to promote general health and well-being, and quality of life of people or by specific protective measures. In disease control, primary prevention aims at protecting the susceptible population who has not yet contracted the disease. WHO (2017) has recommended two major approaches for the primary prevention of diseases where the risk factors are established: Population (mass) strategy and High-risk strategy. Population approach is directed at the whole population irrespective of individual risk levels. It is directed towards socio-economic, behavioral and lifestyle changes. The high -risk strategy aims to bring preventive care to individuals at special risk. This requires detection of individuals at high risk by the optimum use of clinical methods.

Everado, (2013) opined that primary prevention could be individual or population prevention. While the individual primary prevention prevents the disease from occurring and keeps the individual healthy, the population primary prevention tries in maintaining a healthy population and keeps the disease out of the population. Examples of primary prevention include avoidance of sexual promiscuity and use of condom to prevent sexually transmitted diseases (STDs), immunization and vaccination against infectious diseases, washing of hands at appropriate times to prevent contamination and cessation of smoking to prevent lung cancer.

Secondary prevention is action taken to halt the progress of a disease at its incipient stage and prevents complications. The specific interventions include early diagnosis (e.g. screening tests, and case findings) and prompt and adequate treatment before it becomes serious (Yazachew \& Alem). Secondary prevention attempts to arrest the disease 
process, restore health by seeking out unrecognized disease and treating it before irreversible pathological changes take place and reverse communicability of infectious diseases. It thus protects others in the community from acquiring the infection and thus provides at once secondary prevention for the infected ones and primary prevention for their potential contacts. Secondary level prevention tries to minimize the resultant damage after disease occurrence, where primary prevention has failed. It tries to answer the question: once a disease is in a population, how do we control it or move towards its eradication? (Simmon, 2013).

Tertiary prevention seeks to limit disability or complication arising from an irreversible condition (Yazachew \& Alem, 2004). Tertiary prevention is used when the disease process has advanced beyond its early stages. Interventions that are accomplished in the stage of tertiary prevention are disability limitation, and rehabilitation (Ekenedo \& Obiechina, 2016).

Disease control activities usually focus on primary prevention or secondary prevention. However, most successful programmes combine both. Therefore, this discussion shall focus on the role adult education plays in primary and secondary prevention as disease outbreak control measures.

\subsection{Education and Health Behaviour}

Researchers have found that education interventions have the ability of improving health behaviours. For instance,Lam et al. (2004) reported that an intervention programme improved hand washing practice and reduced rates of infection which was sustained 12 months after the intervention. Colombo et al. (2002)) reported that compliance with hand hygiene could be improved by targeted teaching. Though this study was based on an intervention and there was a control group, compliance with the use of alcohol hand rub improved in the control group as well as in the intervention group. The group receiving education did demonstrate a higher level of use of alcohol hand rub. Perkins et al. (2009) also combined interventions, including education, and reported an increase in compliance with infection control precautions and a reduction in MRSA -Methicillin-restant Staphil ococcus Aureus infections. A prospective observational study was undertaken by Trick et al.'s (2007)over a 3 year period. The intervention included education, the introduction of alcohol hand rub and a poster campaign. The authors reported that there was increased adherence to hand hygiene recommendations in the 3 intervention sites when compared with the control site which did not participate in the education or poster campaign. Panhotra et al. (2004) reported that education increased compliance with hand hygiene in nurses as measured by observation. They reasoned that the fact that education was provided continuously rather than episodically as in other studies may have impacted on the findings. Atack and Luke (2008) carried out a quasi-experimental study involving 76 health care professionals including nurses. They reported that education via an online course increased staff's perceptions of their own infection control competence

\subsection{Health Education and Primary Prevention of Diseases}

Like earlier stated, disease control usually focus on primary and secondary prevention. In primary prevention, efforts are made to prevent the onset of disease. Health education has a very important role to play in preventing and delaying the onset of diseases, and in the spread of an already existing disease to vulnerable individuals (Ekenedo \& Obiechina, 2016). Health education targets the population with health information that will equip them with the pre-requisite knowledge for preventing the onset of a disease. It signifies intervention in the pre-pathogenesis phase of a disease or health problem. Primary prevention may be accomplished by health education designed to promote general health and well-being, and quality of life of people or by specially packaged programmes providing information about the causes, signs and symptoms, mode of transmission, and preventive measures regarding the disease in question.

The high -risk strategy aims to bring preventive care to individuals at special risk. This requires detection of individuals at high risk by the use of clinical methods before they can be reached with health education. Education interventions at this level of prevention of chronic diseases can also happen as formal education, non-formal education and informal education. Under the non-formal structure, education interventions that promote healthy lifestyle can be delivered in the workplace, community and hospital settings. Training and building skills in stress management, making healthy food choices, hand washing, personal and environmental hygiene and in recreational activity will build individual self-efficacy which is a major factor in health behavior change. Informal education that can enhance healthy lifestyle comes from our everyday interaction such as health information gathered from friends, relatives, mass media, internet websites, and social networks.

\subsection{Health Education and Secondary Prevention}

The specific interventions in secondary prevention are: early diagnosis (e.g. screening tests, and case findings) and adequate treatment. Secondary prevention attempts to arrest the disease process, restore health by seeking out unrecognized disease and treating it before irreversible pathological changes take place and reverse communicability of infectious diseases. It thus protects others in the community from acquiring the infection and thus provides at once secondary prevention for the infected ones and primary prevention for their potential contacts. Education interventions at this level of prevention can occur as patient education, counseling, workplace and community health education, and informally from information gathered from sources such as books and other printed materials, electronic media and internet. Education exposes the patient with any form 
of disease to information on the nature of the disease - the signs and symptoms, available test, treatment and management options, and the need to adhere to treatment as well as the consequences of not adhering to treatment.

Health education interventions build the capacity of chronic disease patients in carrying out self management of their chronic condition so that disease control increases, health care costs go down, and family well-being improves. For instance, a diabetic patient who understands the physiological explanation of diabetes, signs and symptoms and how to prevent crisis situation would be able to control the condition through diet, blood sugar check and even administer insulin without requiring a visit to a health facility unlike one who is totally dependent on the health personnel for the control of the condition because he or she lacks knowledge. Similarly, health education exposes patients with communicable diseases to the signs and symptoms, causative agents, mode of transmission and preventive measures so that they manage themselves and their environment in such a way that they neither re-infect themselves nor infect persons close to them. For instance, a patient with conjunctivitis who through health education understands that conjunctivitis is spread by direct contact with the infected person or objects such as handkerchief used and contaminated by the infected person would be able to avoid infecting others and re-infecting self by maintaining high personal hygiene and minimizing or avoiding direct contact with persons especially shaking of hands, without feeling rejected.

\subsection{Effective Health Education Methods for Control of Disease Outbreak}

The concept of health education, which now embraces a wider notion of health promotion and a new emphasis on prevention, is increasingly focused on learning and empowerment (WHO, 2005). New methods of teaching in health education have gained ground in accordance with concepts of learning in adult education. Within adult education, the major changes reflect the recognition that adult education has high relevance to current societal issues of which disease outbreak prevention is one. As a result of these changes, new strategies have been developed in areas where health and adult education overlap.

Tennat and Pogson (2004) noted that the various adult learning styles include Visual, Auditory and Kinesthetic, which all require different approaches to presenting information in order to achieve maximum retention. There is a wide variety of methods that can be used in health educating adults. However, the choice of method will be determined by factors such as age of the participants, educational level of the participants, the topic of instruction, availability of Information, Education and Communication (IEC) materials and expertise of the health educator. Most widely used methods both in formal and nonformal settings include: health talks, group discussion, demonstration, lecture, games, role play, song, question and answer, guest speaker, visits/ excursion. Most times a combination of two or more methods produce the best results. Some of the common ones are hereby discussed.

\subsection{Health Talks}

Talking is often the most natural way of communicating with people to share health knowledge and facts. However, talks are most effective if conducted with small gatherings (5-10 people), because the larger the group the less chance that each person has to participate (Ethiopian Federal Ministry of Health, 2011). Talking to a person who has come for help is much like giving advice. But advice is not the same as health education. To make a talk educational rather than just a chat, it will beneficial if it is combined with IEC materials, especially visual aids, such as posters and flyers or audiovisual material. Also a talk can be tied into the local setting by the use of proverbs and local stories that carry a positive health message. Health talk can take place in several settings such as the hospital, school, market, workplace, church, community hall, and even in a bus. Delivering a health talk efficiently requires the health educator to know the audience; be well prepared; and practice adequately.

\subsection{Demonstration}

The use of this method in health education is based on learning through observation. There is a difference between knowing how to do something and actually being able to do it. The aim of a demonstration is to help learners become able to do the skills themselves, not just know how to do them. The adult health educator should be able to find ways to make health related demonstrations a pleasant way of sharing skills and knowledge. 'Showing how is better than telling how' since a Chinese proverb says: 'If I hear, I forget; If I see, I remember; If I do, I know. Demonstration makes learning faster and surer. A typical example of using this method in providing adult education during epidemic is demonstration of proper hand washing as a way of controlling the spread of Ebola virus.

\section{Group Discussion}

Group discussion involves the free flow of communication between a health educator and two or more participants. Often a discussion of this type is used after a slide show or following a more formal presentation. This type of teaching method is characterized by participants having an equal chance to talk freely and exchange ideas with each other. In most group discussions the subject of the discussion can be taken up and shared equally by all the members of the group. In the best group discussions, collective thinking processes can be used to solve problems. These discussions often develop a common goal and are useful in collective planning and implementation of health plans. This method is effectively used with people suffering from a common health problem such as People Living with HIV/ AIDS, TB patients and diabetics. Under group discussion they can share experiences and identify better ways of dealing with their conditions. It can equally be effective when providing 
health education to people who are gather together for a common purpose that presents the opportunity for health education such as the workplace and during antenatal clinics.

\subsection{IEC Materials for Effective Adult Health Education}

Health learning materials that can be used in health education during disease epidemic are usually broadly classified into four categories: printed materials, visual materials, audio and audio-visual materials.

\subsection{Printed Materials}

Printed health learning materials can be used as a medium in their own right or as support for other kinds of media. Some printed health learning materials that are common include posters, leaflets and flip charts.

\subsubsection{Posters}

In recent years, the use of posters in communicating health messages has increased dramatically (Ethiopian Federal Ministry of Education, 2007). Since a poster consists of pictures or symbols and words, it communicates health messages both to literate and illiterate people. It has high value to communicate messages to illiterate people because it can serve as a visual aid. The main purposes of posters are to reinforce or remind people of a message received through other channels, and to give information and advice. They also function to give directions and instructions for actions, such as a poster about proper hand washing procedure. Posters can also serve to announce important events and programmes such as World HIV/ AIDS Day. Visual aids like posters explain, enhance, and emphasize key points of health messages. They allow the audience to see your ideas in pictures and words, hence, making learning surer.

\subsubsection{Flip chart}

Flip charts are useful to present several steps or aspects that are relevant to a central topic, such as, demonstration of the transmission of cholera. When flip chart is used in health education each page must be discussed completely before turning to the next. At the end it is necessary to go back to the first charts to review the subject and help people remember the ideas. Flip chart can be used to health educate individuals and groups in the hospital, workplace and community settings.

\subsubsection{Leaflets}

Leaflets are the most common way of using print media in adult health education. They can be a useful reinforcement for individual and group sessions and serve as a reminder of the main points that have been made. They are also helpful for sensitive subjects such as HIV/ AIDS and other sexually transmitted infections. When people are too shy to ask for advice they can pick up a leaflet and read it privately. In terms of content, leaflets, booklets or pamphlets are best when they are brief, written in simple words and understandable language. It is usually relevant to include address at the back to indicate where people can get further information. One of the advantages of leaflet is that it is not bound by time and location. Leaflets can be given out anywhere and anytime.

\subsection{Visual Materials}

Visuals materials are one of the strongest methods of communicating health messages, especially where literacy is low amongst the population. They are good when they are accompanied with interactive methods. It is said that a picture tells a thousand words. Real objects and video do the same. They are immediate and powerful and people can play with them.

\subsection{Audio and Audio-Visual Materials}

Audio material includes anything heard such as the spoken word, a health talk or music. Radio and audio cassettes are good examples of audio aids. During disease outbreaks the radio usually plays very significant role in disseminating information to the general public. Announcements, jingles, discussions and drama are different ways the radio are used to communicate and educate people on diseases that are trending.

As the name, implies, audio-visual materials combine both seeing and listening. These materials include TV, films or videos which provide a wide range of interest and can convey messages with high motivational appeal. Audio-visual health learning materials can arouse interest if they are of high quality and provide a clear mental picture of the message. They may also speed up and enhance understanding which hastens action. Drama series on TV and films on DVD can be used to communicate information about diseases and health problems that are emerging and spreading but are not emergency situations. They can equally deliver enlightenment on how to behave during infectious disease outbreaks.

Electronic media whether audio or audio-visual remains the fastest means of communicating health information during a disease outbreak. It has the unique ability of reaching a wide target audience at a time. This media is particularly useful when the disease involved is acute and deadly in nature such as Ebola, Lassa fever, Bird Flu and Anthrax in which case there is urgent need to reach a large population with health information as quickly as possible. 


\section{The Use of Technology Tools in Adult Health Education for Chronic Diseases Control}

In this age of high technology, there are a lot of innovative ways of applying educational technology tools in communicating health information for speedy and wider reach of target adult audience especially during disease outbreak. Computer and internet literacy is growing fast. Some people can benefit from these technologies because ownership of personal computer (PC), smart phones and internet connections is fast growing among urban dwellers, though rural dwellers may be seriously handicapped as a result of poverty and lack of electricity. Nevertheless, this communication strategy offers a big boost to information communication during disease outbreak ensuring speedy and wider coverage of the public in a very short time.

The internet contains information about almost all diseases and serves as a source of information to a number of adults who have access to it. Information concerning health matters can be downloaded, or printed by individuals. However, one of the major drawbacks of health information in the internet is the fact that the credibility of some of the information posted in the internet cannot always be guaranteed. The public consume false and misleading health information which can be damaging to their health.

During communicable disease outbreak, health information can be communicated effectively through the use of hand phone. Social media platforms such as 'WhatsApp', 'Facebook', and SMS are veritable media for conveying information about the nature of the disease in question: the causes, signs and symptoms, mode of transmission and preventive measures. The social media is awash with all sorts of health messages posted most times by non health professionals and outright quacks. The negative consequences of proliferation of health messages in social media are enormous. People have been known to meet their untimely death by following and applying information gathered from social media. For example, the myth about drinking and bathing with concentrated salt solution as a means of getting protection from Ebola virus spread like wild fire in Nigeria during the Ebola virus outbreak of 2014 resulting in the unfortunate and avoidable death of some hypertensive patients who acted on such information.

Persuasive technology is another innovation that can be successfully applied in helping people learn and adopt healthy behaviours. This technology is most suitable for chronic diseases rather than acute disease conditions. Persuasive technology is defined as a computer system, device, or application that is intentionally designed to change a person's attitude or behavior (Fogg 2003). This technology uses tools (e.g., balance board), media (e.g., video, audio, or both), and social interaction (e.g., playing with another person) to persuade individuals to adopt positive health behavior without their actually knowing it. One of such persuasive technology media known as Dance Dance Revolution (DDR) which was not developed specifically to promote healthy behaviour has changed exercise attitudes and behavior of people using principles of persuasive technology. Dance Revolution uses video, music, and a dance platform to capture interest and engage people in the activity without their being fully aware that they are exercising. The emerging field of persuasive technology has enormous potential for promoting physical activity and healthy behaviours (Fogg \& Eckles 2007; Zhu 2008) which can reduce the incidences of chronic diseases such as diabetes, cancers and heart diseases.

\section{Constraints to Effective Health Education for Disease Outbreak Control}

Irrespective of the key role education play in the control of disease outbreak, its implementation has not been without challenges.

\subsection{Dearth of Professional Health Educators in Health Care Service}

Health education is lifelong education which means that much of health education happen at adult stage of life. Many higher institutions graduate health educators trained to work in the school setting. Medical personnel in health service delivery did not receive adequate training in the rudiments of health education. Hence, a gap exists in the health care delivery in Nigeria in the area of health education that desperately yearns to be filled.

\subsection{Lack of Infrastructure}

The use of mass media like TV and Radio, and technological tools like hand phones and computer especially in the rural communities in developing countries in the control of disease outbreaks presents a big challenge due to lack of basic infrastructure such as electricity. Most innovative technologies are dependent on electricity for optimal use. The epileptic nature of electricity supply and unreliable telecommunications network even in urban areas is a draw back to the effective use of mass media and technology in communicating health information in Nigeria.

\subsection{Low Level of Computer Literacy}

To be able to benefit maximally from current innovative technologies, there is need for a certain level of literacy in the use of computer. It is true that many Nigerians have access to and use a wide range of information and communication technologies, a larger number are yet to fully benefit from the ICT world especially older adults and those in the rural areas due to lack of skills in the use of computer. In addition, sometimes those who have the responsibility of giving health education are not competent in the use of computer. 


\section{Conclusions}

It has been proven that controlling disease outbreak within a population cannot be effectively achieved without education intervention targeted at both high-risk individuals and the masses. Adults have the responsibility of protecting themselves and their young from diseases and infirmities so health education targeted at them will go a long way in achieving the desired goal of disease outbreak control by equipping them with the necessary information and skills needed to prevent either the onset of the disease or the progression of the disease among the people at risk. This can be achieved through wellarticulated health education programmes, whether formal, non-formal or informal, communicated with the right methods and effective IEC materials as well as innovative technology baring constraints such as dearth of trained health education personnel, incompetency in the use of computer and IEC material and lack of social infrastructure.

\section{Suggestions}

Flowing from the conclusions, the following suggestions are made.

- Health education should be a General Studies course for all formal adult education programmes in Nigeria.

- All forms of adult health education that take place at various non-formal settings should be carried out by trained and qualified personnel.

- All Health professionals who work in health facilities including Doctors, Nurses, and Pharmacists should receive training in health education so that they can effectively perform patient education.

- Institutions of higher learning that offer Adult Education should as a matter of importance have Health Education as a course combination option. If Adult Education offers a combination with Health Education, higher institutions would be able to contribute health education personnel to the health care delivery system that would be responsive in times of disease outbreaks.

- IEC materials should be made available by relevant agencies for effective adult education for the control of disease outbreak.

- Social infrastructures especially electricity should be provided by government in order to achieve effectiveness in the use of technological tools for information dissemination in the control of disease outbreak.

\section{References}

i. Atack, L. \& Luke, R. (2008).Impact of an online course on infection control and prevention competencies. Journal of Advanced Nursing; 63: 175-180.

ii. Colombo, C., Giger, H., Grote, J., Deplazes, C., Pletscher, W., Luthi, R., \& Ruef, C. (2002)Impact of teaching interventions on nurse compliance with hand disinfection. Journal of Hospital Infection; 51: 68-72.

iii. Ekenedo, G. O. \& Obiechina, G. O. (2016). Reducing the burden of chronic diseases in Nigeria through health education. Nigerian School Health Journal; 28, (2), 118 - 133.

iv. Ethiopian Federal Ministry of Health (2011). Health education, advocacy and community mobilization, part 1: Blended learning module for health extension programme. Ethiopia: Federal Ministry of Health. www.open.ac.uk/ africa/ heat

v. Everado, V. (2013). Division of viral diseases, Center for Disease Control and Prevention.

vi. Fogg, B. J. (2003)Persuasive Technology: Using Computers to Change what We Think and Do. San Francisco: Morgan Kaufmann publishers.

vii. Fogg, B. J., \& Eckles, D. (2007). Mobile Persuasion: 20 Perspectives on the Future of Behavior Change. Stanford: Stanford Captology Media.

viii. Hedi, S. (2015). National Center for Immunization and Respiratory Diseases. USA: Center for Disease Control and Prevention.

ix. Lam, B.C.C., Lee, J., \& Lau, Y.L. (2004).Hand hygiene practices in a neonatal intensive care unit: a multimodal intervention and impact on nosocomial infection. Pediatrics; 114: 565-571.

x. Merriam, Sharan, B. \& Brockett, Ralph, G. (2007). The Profession and Practice of Adult Education: An Introduction. Jossey-Bass.

xi. Panhotra, B.R., Saxena, A.K., Al-Ghamdi, A.M., \& Al-Arabi. (2004). The effect of a continuous educational program on hand washing compliance among healthcare workers in an intensive care unit. British Journal of Infection Control; 5: 15-18.

xii. Peterson,D.(2017).Whatisadulteducation. http:/ / adulted.about.com/od/ whatisadultlearning/ p/ whatisadulteducation.htm

xiii. Simmons, K. (2013). Duration of immunity to norovirus gastroenteritis. Emerging Infectious Disease, 19(8): p. 12607.

xiv. Tennant, M. \& Pogson, P. (2004). Learning and Change in the Adult Years: A Developmental Perspective. San Francisco: Jossey-Bass Publishers.

xv. The Canadian Encyclopedia (2014). "Adult Education"..http:// www.thecanadianencyclopedia.ca/ en/article/ adulteducation/

xvi. Williamson, D. L. \& Carr, J. (2009). Health as resources for everyday life. Advancing the conceptualization. Journal of Critical Public Health; 19, (1); 107-122. 
xvii. WHO (2005). The WHO STEP- wise approach to chronic disease risk factors surveillance. WHO STEPS Surveillance Manual. Geneva: World Health Organization.

xviii. WHO (2006). The impact of chronic disease in Nigeria.www.who.int/ chp.chronic disease report/ en/

xix. WHO (2017). Disease Outbreak.http:/ / www.who.int/ topics/ disease outbreaks/ en/

xx. Yazachew, M. \& Alem, Y. (2004). Introduction to Health Education. Ethiopia: Ethiopia Public Health Initiative.

xxi. Zhu, W. (2008). Promoting physical activity using technology. Research Digest series 9, no 3.Washington DC: President's Council on Physical Fitness and Sport 